\title{
An improved method for determining the flow characteristics of prosthetic mitral heart valves
}

\author{
J. T. M. WRIGHT and L. J. TEMPLE \\ Bio-engineering and Medical Physics Unit, Department of Surgery, University of Liverpool and the \\ Cardio-thoracic Surgical Centre, Broadgreen Hospital, Liverpool
}

\begin{abstract}
The flow characteristics of most prosthetic mitral valves recommended for clinical use have not been adequately investigated. As a result vital information about their performance is lacking and, until this is published, comparisons between different prostheses cannot easily be made. In this paper the design and construction of a suitable rig for the testing of such valves is described. The results obtained will be presented in a subsequent paper.
\end{abstract}

Since the introduction of heart valve replacement as an accepted clinical procedure (Starr, 1960 ; Cartwright, Palich, Ford, Giacobine, Zubritsky, and Ratan, 1962 ; Barnard, Goosen, Holmgren, and Schrire, 1962) the number of valve prostheses available to the surgeon has steadily increased, and his choice of prosthesis has consequently become more difficult.

The follow-up of patients having such valves fitted has made the deficiencies of the prostheses clear. These deficiencies include clot propagation and emboli, infection, valve wear, malfunction, incompetence, and excessive pressure gradient. The surgeon's choice of prosthesis would have been aided by published measurements of valve characteristics comparing different types, but these tests have not been carried out over a suffciently wide range of operating conditions to be informative. As a consequence many new valves have been designed empirically and data on their essential characteristics are lacking.

It is accepted that ultimately every new valve must undergo biological testing, but observation of the valve under conditions simulating that of the human circulation enables measurements to be made that cannot be made in biological experiments. Since the technical problems of mitral and aortic replacement are different, and as aortic valve replacement has been clinically more successful than that of the mitral valve, this work is confined to the study of mitral valve prostheses.

\section{PREVIOUS METHODS OF TESTING}

Two basic methods of testing have been used in the past: (a) using a heart, whether living or dead. Internal and external pulse duplicators fall into this group (Calvert, Drabble, Serafin and Temple, 1964 ; Duran, Gunning, and McMillan, 1964) ; and (b) using some form of mechanical rig (Wieting, 1968).

The defects of internal pulse duplicators are well known. Our preliminary experiments with an external pulse duplicator led to the conclusion that although biological pulse duplicators are useful for observing the action of natural valves, they are not suitable for the scientific assessment of prostheses.

The mechanical rigs used in the past have shown considerable variation in design and performance which makes it difficult to compare the results of tests of different prostheses. Many workers have not specified the viscosity of the fluid used (Beeson, West, and Burns, 1965), others have used water (Steinmetz, May, Mueller, Anderson, and Merendino, 1964), and a few a blood analogue (Smeloff, Huntley, Davey, Kaufman, and Gerbode, 1966). The shape of the flow passages has not often been considered and the instantaneous flow-time characteristics have not been standardized.

\section{DESIGN CONSIDERATIONS}

In the design of the method the following specifications were laid down:

(a) The fluid used for testing should be a blood analogue, i.e., the viscosity and density should be similar to blood at $37^{\circ} \mathrm{C}$.

(b) The flow characteristics should be known, should approximate to physiological flow, and should be reproducible. 
(c) The flow passages of the pulse duplicator should resemble as closely as possible the vessels and chambers of the normal heart.

The manner in which these specifications have been met is discussed below.

THE BLOOD ANALOGUE The outstanding characteristic of blood is that it is composed of a suspension of red cells in a fluid. The suspension makes blood non-homogeneous in character and therefore non-Newtonian. As a result blood shows anomalous viscous properties when flowing in small vessels, particularly capillaries, and also when flowing at very low shear rates. In the large passages of the heart, however, blood may be considered a Newtonian fluid, i.e., it behaves like homogeneous fluid and not a suspension of particles.

The viscosity changes which are shear dependent were discussed by Taylor (1959) with regard to pulsatile flow, and he demonstrated that the value of viscosity at high shear rates was clearly the best one to take. This being so, it is necessary to make any viscosity measurements of blood using a viscometer at a high shear rate. Published data showed considerable variation in viscosity (McDonald, 1960) and therefore some measurements were made. Heparinized blood samples were taken from patients undergoing open-heart surgery. The viscosity of each sample was measured at several different temperatures using a Ferranti rotating cylinder viscometer at shear

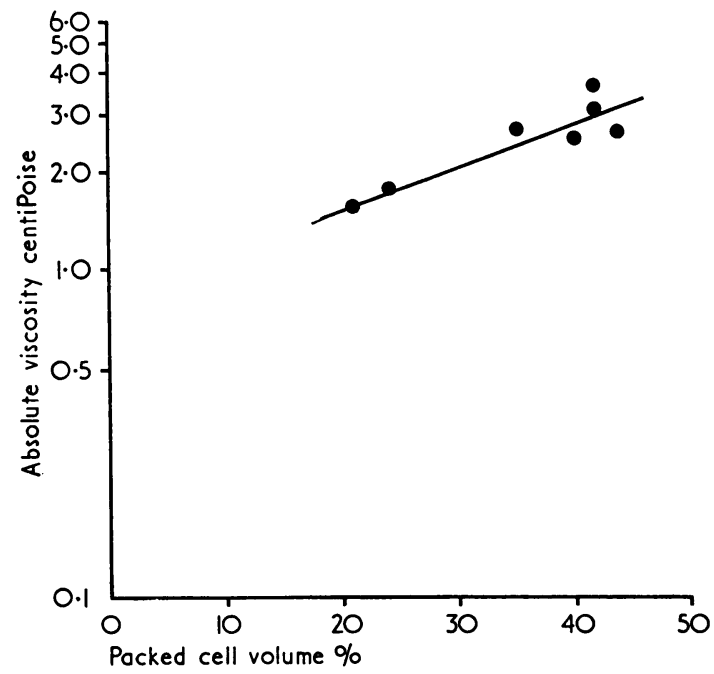

FIG. 1. Variation of viscosity, with packed cell volume of fresh human blood at $37^{\circ} \mathrm{C}$. rates of $628-950 \mathrm{~s}^{-1}$; the haematocrit was deter $-\frac{\vec{F}}{-}$ mined by the usual clinical method.

The results obtained are summarized in $\frac{\bar{c}}{\bar{s}}$ graphical form in Figure 1. A value of the normal $\frac{\infty}{\sigma}$ haematocrit was taken at $45 \%$ (Geigy, 1956) and $\cong$ so from the graph the mean viscosity obtained $\%$ was $3.0 \mathrm{cP}$.

A water glycerine mixture which had a vis- $\stackrel{-}{-}$ cosity of $3.0 \mathrm{cP}$ at the working temperature of $\vec{\omega}$ $25^{\circ} \mathrm{C}$ was therefore used for the tests. This solution had a density of 1.083 .

THE FLOW CHARACTERISTICS The factors which were considered important to the flow of the $\vec{\circ}$ blood analogue through the prosthetic valve $\vec{\circ}$

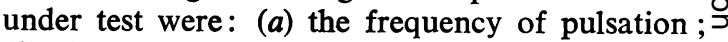
(b) the volume displacement per pulsation; and $\vec{c}$ (c) the volume-time characteristics.

The frequency of pulsation was made variable over the range $60-180$ pulses per minute by driving the rig with a variable speed DC motor. The $\vec{\varphi}$ range chosen corresponded to normal physiological limits.

The volume displacement per pulsation in the normal physiological situation varies according to the demands of the body over the range of about 60-180 ml. In order to simplify the rig construc- $\frac{0}{\mathrm{D}}$ tion and calibration, three separate stroke $\cong$ volumes were chosen. The stroke volumes used $\overrightarrow{\overrightarrow{0}}$ were 58,126 , and $184 \mathrm{ml}$ per stroke. This pro- 3 vided a simulated cardiac output varying from $3 \cdot 5$ to 30 litres per minute.

We decided to adopt a sine wave function for the volume displacement of the rig, rather than try to reproduce the physiological volume-time $x$ characteristics. The reasons were as follows:

(a) The ventricle of the heart is not able to produce a negative pressure and a suction effect 0 (Henderson, 1906). The flow of blood into the ventricle must therefore be a function of the $\frac{\text { 의 }}{8}$ pressure in the atrium and the gradient across the $\frac{}{0}$ mitral valve. It follows that the filling rate of the ventricle will be dependent upon the pressure $N$ gradient-flow characteristics of the mitral valve, whether it be normal, diseased or a prosthesis. Thus a precise simulation is not possible.

(b) It is mechanically simple to produce and reproduce a sine wave. Results of tests by different $\stackrel{\varrho}{O}$ workers in different institutions will therefore be $\mathbb{D}$ comparable.

(c) Although physiologically the diastolic and 0 systolic periods are dissimilar, as only the mitral $\frac{\vec{d}}{\mathbb{D}}$

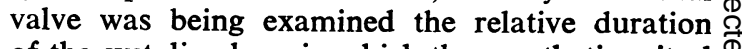
of the systolic phase in which the prosthetic mitral $\stackrel{\mathbb{\Omega}}{\varrho}$ valve remained closed was not of great importance. 
THE FLOW PASSAGES In order to retain the anatomical shape of flow passages and mechanical simplicity we made a rigid Perspex model of the left ventricle and atrium. To determine the correct shape a number of plaster-of-Paris casts of the flow passages of human and pig hearts were made. During casting the coronary arteries were perfused with saline at $100 \mathrm{mmHg}$ in order to distend the heart into its physiological shape. These casts were measured, and, from the mean dimensions found, a Perspex model of the left atrium and ventricle (Fig. 2) was constructed.

The atrium was made detachable so that the test valve could conveniently be changed, and the ventricle was made of fixed size for mechanical simplicity.

FUNCTION OF THE RIG Fluid was fed by four tubes to the atrium from the atrial reservoir. The tubes were each $1 \mathrm{~cm}$ bore and represented the pulmonary veins.

A pipe was connected from the apex of the ventricle to a piston pump. When the piston receded in the cylinder, fluid was drawn from the atrial reservoir, through the mitral valve under test, and hence into the ventricle. When the piston advanced in the cylinder the fluid displaced was ejected from the ventricle through an outlet pipe which represented the aorta. The pipe contained a spring-loaded non-return valve which would open when the pressure in the ventricle exceeded 100 $\mathrm{mmHg}$. The circuit diagram of the rig is shown in Fig. 3 and a photograph of the rig in Fig. 4.

The piston pump had two pistons of differential diameters connected in tandem to a common connecting rod and reciprocated in a steppedbore cylinder. The piston diameters chosen were such that the larger piston displaced about 180 $\mathrm{ml}$ per stroke, and the smaller about $120 \mathrm{ml}$ per stroke. Two gate valves determined the volume displaced by the pump which was 58,126 , or 184 $\mathrm{ml}$ per stroke, depending on the position of the valves. The pistons were actuated by a mechanical sine wave generator, the stroke displacement of this machine was approximately $28 \mathrm{~mm}$, and it was driven by a variable speed DC shunt motor.

In order to keep the temperature and therefore the viscosity of the fluid constant during a test run a thermostatically controlled bath was used as the main fluid reservoir maintaining the temperature at $25 \pm 0.5^{\circ} \mathrm{C}$.

A circulating pump was used to pump fluid from the bath into the left atrial feed reservoir, and the level was maintained in this by a simple overflow. The excess fluid was run under gravity back into the bath. To prevent aeration it was necessary to feed the overflow into a section of the bath containing part of the debubbling section

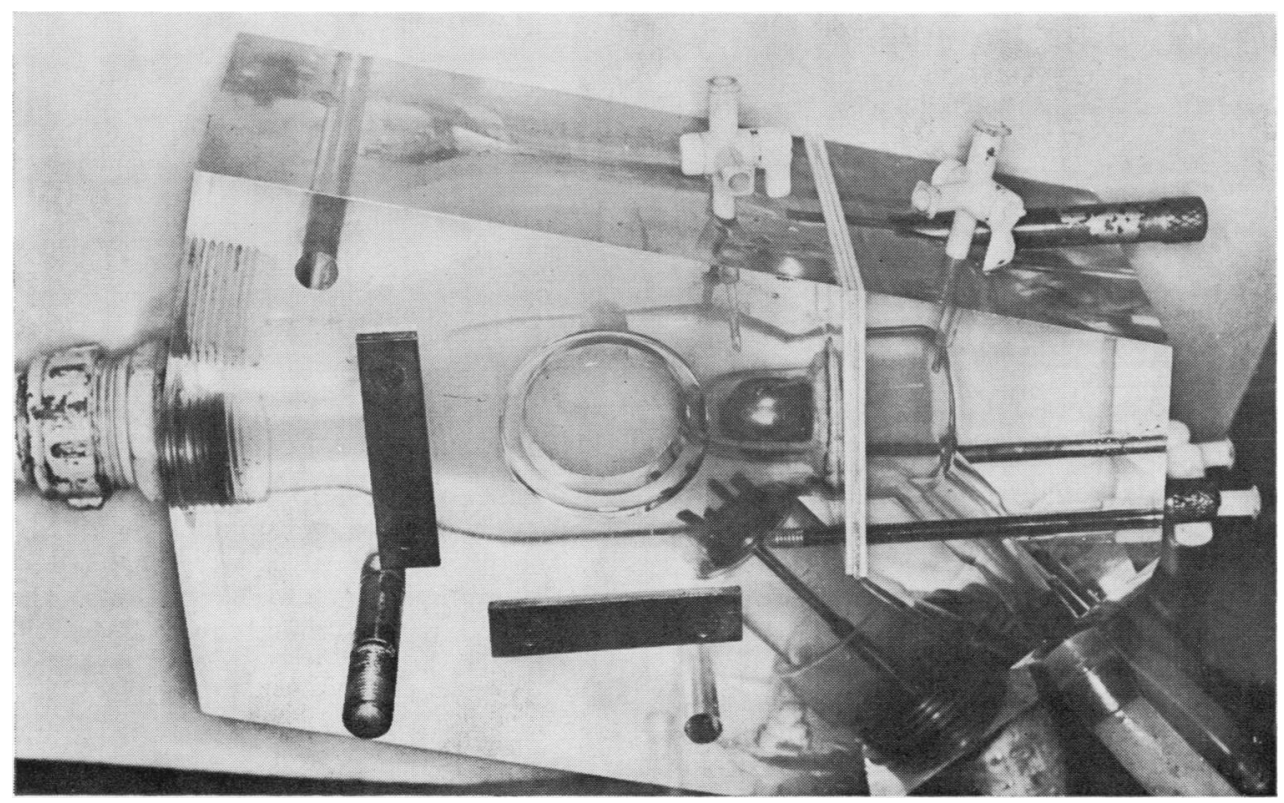

FIG. 2. Perspex model of left atrium and ventricle with anemometer probe and cover removed. 


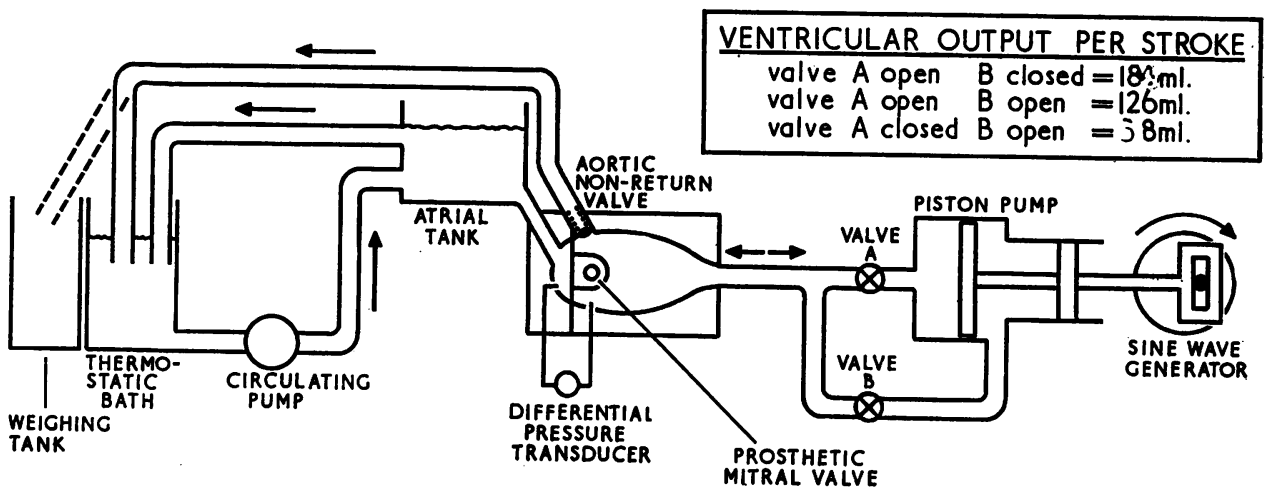

FIG. 3. Schematic diagram of rig pulse duplicator.

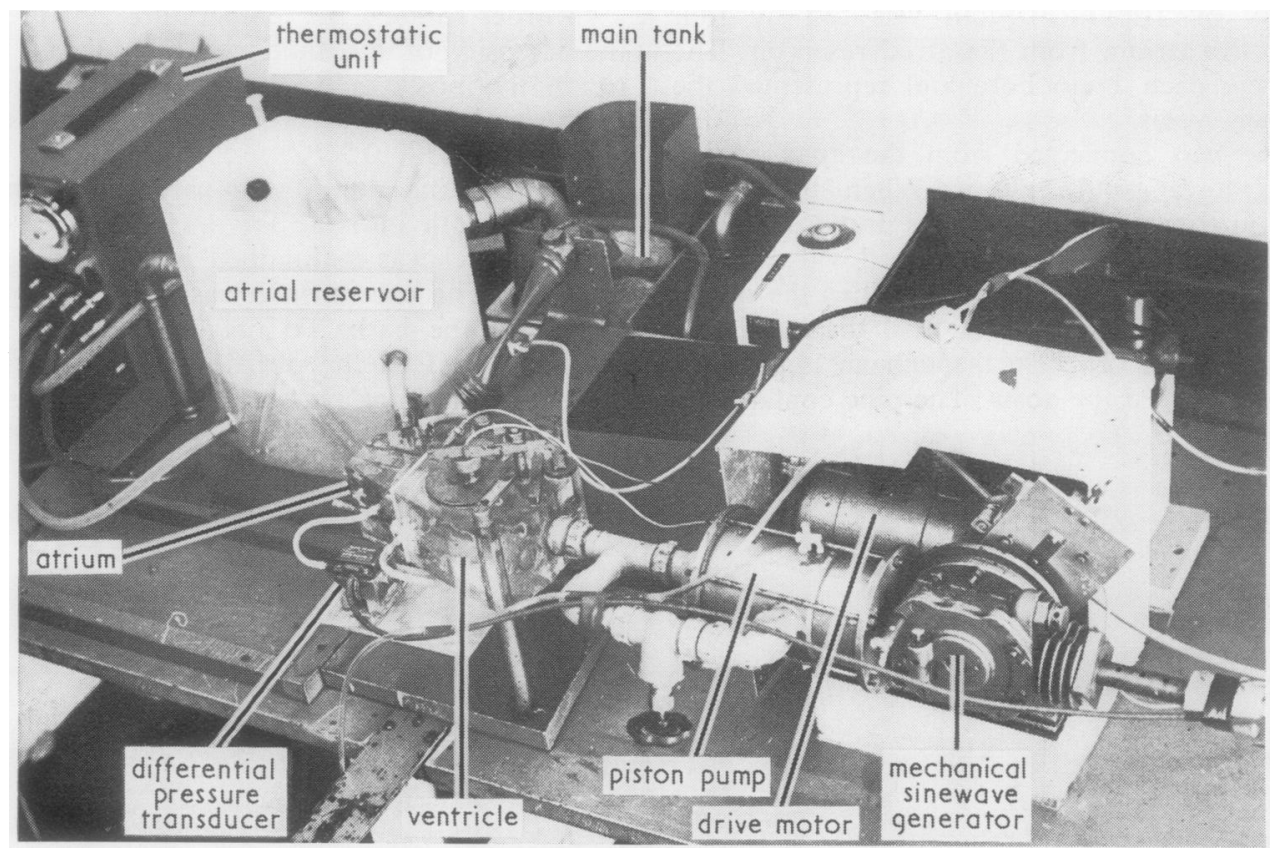

FIG. 4. View of rig pulse duplicator.

from a Rygg oxygenator, which was held in position by a fine gauze plate. The outlet pipe from the ventricle directed the ejected fluid back into the bath, but, when required, the fluid could be collected in a separate tank to be weighed. The actual volume displacements of the piston pump were measured under static conditions and the displacement of the piston pump was measured under dynamic conditions (the stroke of the pump was found to increase slightly with increased running speed).
TEST PROCEDURE Before the valves could be $\overbrace{\omega}^{N}$ tested, they had to be suitably mounted in the 0 rig. The prosthetic valves were each attached to a flat aluminium plate, $1.5 \mathrm{~mm}$ thick. The plate was pierced with a round hole, larger than the $\stackrel{\infty}{+}$ valve orifice, but small enough so that the orifice 0 body could rest against the plate. The hole, chamfered on the atrial side, was surrounded by a ring $\stackrel{\mathbb{D}}{\stackrel{\mathrm{D}}{ }}$ of $1 \mathrm{~mm}$ diameter holes about $3 \mathrm{~mm}$ apart, $\mathbb{\mathbb { D }}$ through which the valve was sutured. Leakage $\frac{\circ}{\sigma}$ around the sewing ring was prevented by sealing 

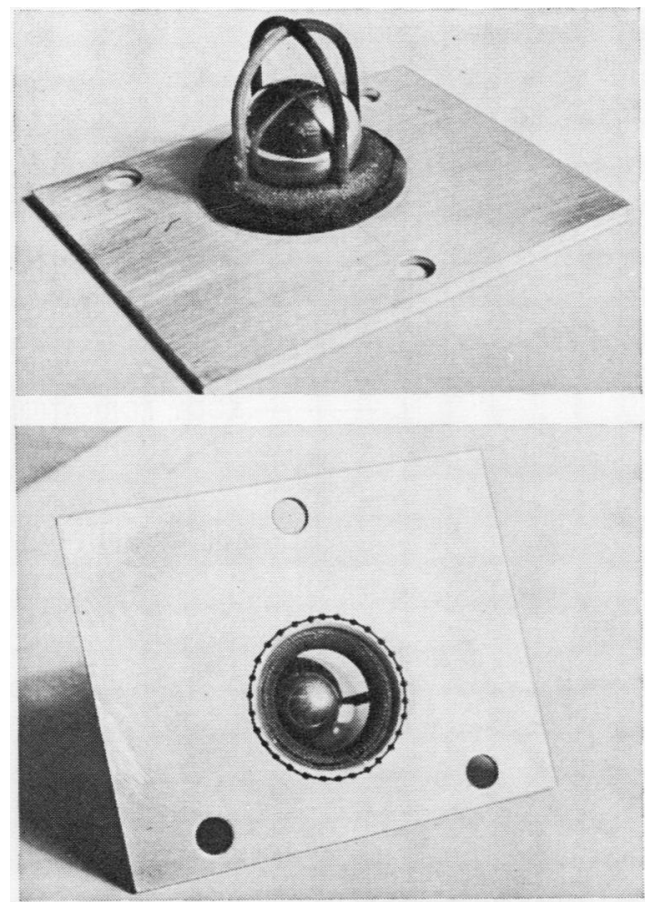

FIG. 5. A typical valve attached to its mounting plate.

it with a self-curing silicone rubber compound. Figure 5 shows a typical valve attachment.

Each plate could be located between the atrial and ventricular sections of the pulse duplicator. The valves, mounted in this manner, could quickly and easily be interchanged.

The rig was prepared for testing by filling it with the water-glycerine mixture. The circulating pump was then started, the temperature adjusted to $25^{\circ} \mathrm{C}$, and the viscosity of the blood analogue checked. Deviation from the chosen viscosity of
$3.0 \mathrm{cP}$ was corrected by adding water or glycerine (the tolerance used was $\pm 1.5 \%$ ). Air was bled from the ventricular cavity and the cylinder(s) and the drive motor were started. The rig was run for several minutes to give time for conditions to stabilize and was then stopped. The viscosity and temperature were then rechecked and readjusted if necessary. The tests carried out are described below.

THE FLOW MEASUREMENTS The flow measurements that were required on the valve under test were:

(a) the mean and instantaneous diastolic pressure gradient ;

(b) the incompetence of the valve ;

(c) observation of the valve action by direct vision and ciné photography ; and

(d) a measure of the flow velocity and turbulence.

Measurement of mean diastolic pressure gradient The diastolic pressure gradient of the valve under test was measured by an ElectroMechanisms $\pm 35 \mathrm{kN} / \mathrm{m}^{2}$ differential pressure transducer. The transducer calibration was checked at the start and conclusion of each test.

The rig speed was adjusted in turn to 60,80 , 100,120 , and 140 pulses per minute, and at each step the output signal from the transducer amplifier was monitored on an oscilloscope. The time base of the oscilloscope was synchronized to the rig cycle and the controls were adjusted so that the zero gradient level was in the lower half of the screen, and the diastolic pressure gradient signal displaced the trace vertically upwards by a convenient amount.

During systole the pressure gradient across the mitral valve, shown in Fig. 6a, was $-105 \mathrm{mmHg}$ (this was because the negative pipe of the transducer was connected to the ventricle, and the
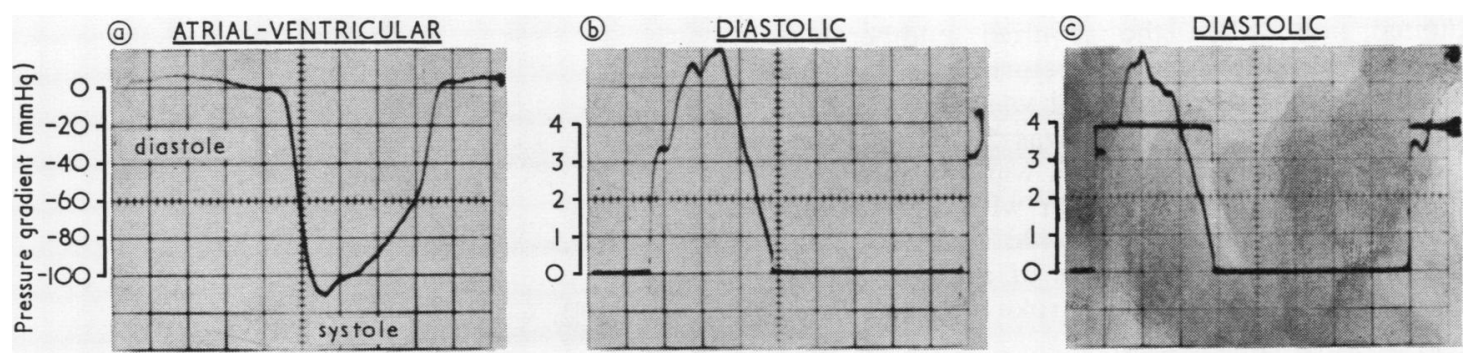

FIG. 6. (a) Atrial-ventricular pressure gradient across mitral valve prosthesis during rig cycle;

(b) Amplified diastolic pressure gradient across mitral valve prosthesis with systolic pressure svppression; (c) As Fig. 6b but with switched DC voltage equal to the mean value superposed. 
systolic ventricular pressure was $105 \mathrm{mmHg}$ ). The mean diastolic pressure gradient was the mean value of the instantaneous gradient over the diastolic phase of the rig cycle. In order to obtain this mean value the systolic portion of the waveform had to be suppressed. To achieve this systolic suppression a cam-operated micro switch was used. The cam was coupled to the drive shaft of the piston pump, and as the shaft rotated it caused the micro switch to turn the signal off during systole, leaving the diastolic gradient signal intact as shown in Fig. 6b. This signal was fed to an integrating amplifier and the output of the integrator was adjusted to give almost full-scale deflection on an output meter. When the meter had settled down to give a steady deflection the signal from the pressure transducer was interchanged for a steady voltage, which was fed to the micro switch in its place.

The voltage was switched on and off for the same length of time as the transducer output had been. The DC level of the applied voltage was then adjusted so that the integrator output meter read the same as before. The voltage being applied to the switch was then measured by means of the digital voltmeter, and this level was equal to the mean diastolic gradient (Fig. 6c), i.e., the area under both curves was the same.

MEASUREMENT OF VALVE INCOMPETENCE The incompetence of the valve was determined by comparing the quantity of fluid ejected from the ventricle for each stroke of the piston with the previously determined volume displaced by the piston. The difference between these two values was due to retrograde flow through the mitral valve. Clearly some retrograde flow is always needed to close a valve.

The rig was started and the required speed set. The aortic outlet tube was transferred from the bath so that the ejected liquid was collected in a weighing tank. The systolic ejections were counted, and when the required number was reached, the outlet tube was returned to the bath. The number of ejections used was 100 when the stroke volume of the pump was $58 \mathrm{ml}$, 40 when it was $126 \mathrm{ml}$, and 25 when it was $184 \mathrm{ml}$.

The tank was weighed both with and without the collected fluid. The specific gravity of the fluid was measured by means of an hydrometer, and the output ejected per stroke was calculated from the formula:

Ventricular output per stroke, Vv (ml)

$$
=\frac{\mathrm{W} \times 1000}{\mathrm{~N} \times \text { sp. gr. } \times 0.998}
$$

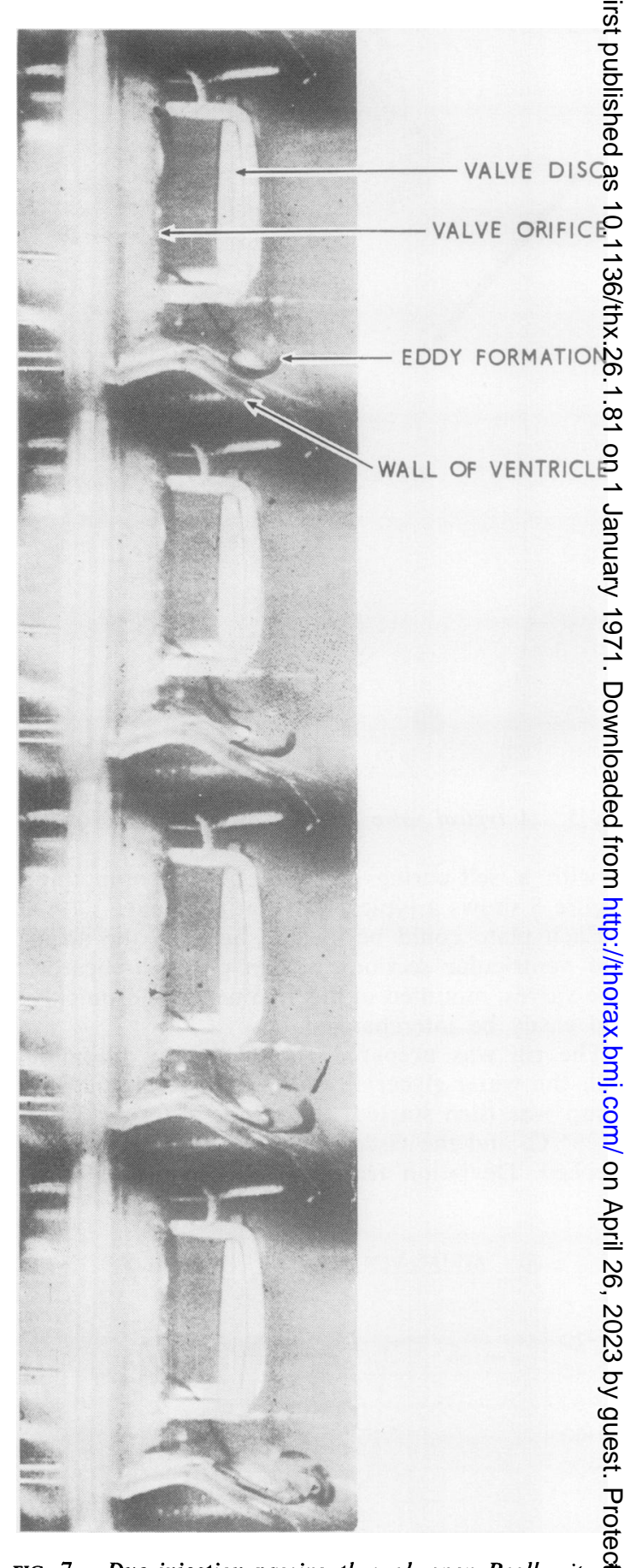

FIG. 7. Dye injection passing through open Beall mitra帖 valve showing eddy formation distal to the disc. Photo graphed at 250 frames per second. 
where $\mathrm{W}$ is the weight of liquid collected $(\mathrm{kg})$, $\mathbf{N}$ is the number of systolic ejections, sp. gr. is the specific gravity of liquid, and 0.998 is the density of water at $25^{\circ} \mathrm{C}$.

The incompetence of the valve, expressed as a percentage of the stroke volume displacement of the pump, was calculated from

$$
\text { Incompetence } \%=\frac{(\mathrm{Vp}-\mathrm{Vv}) \times 100}{\mathrm{Vp}}
$$

where $V p$ is the stroke volume displacement of the pump (ml) and $\mathrm{Vv}$ is the ventricular output per stroke $(\mathrm{ml})$.

VISUALIZATION OF VALVE MOVEMENT In order to record and study the mechanical movements of valves during pulse duplication, $16 \mathrm{~mm}$ cinephotography was used. An H 16 Bolex reflex camera was employed for taking film at 24 and 64 frames per second and a Fastex high-speed camera at 250 and 500 frames per second. The light source was a $650 \mathrm{~W}$ quartz iodine lamp mounted immediately above the lens. The film used was Kodachrome II or high-speed Ektachrome ; both cameras were fitted with extension tubes. The Bolex was mounted on special mounts, and the Fastex on its own heavy stand. The correct lens apertures were found by trial and error.

The cameras recorded atrial and lateral views of the valves. When the lateral view (and with the Fastex and atrial one also) was being photographed, the image of the valve was reflected by a surface aluminized mirror placed at $45^{\circ}$. The camera could then be used in the normal horizontal plane. Valves were filmed with one or both cameras as required (Fig. 7).

The flow patterns through and distal to the valve were visualized by injecting dye into the valve orifice, while the valve action was recorded with the Fastex camera. Naphthalene black in a water glycerine mixture of $3.0 \mathrm{cP}$ viscosity was injected during diastole, $0.25 \mathrm{ml}$ at a time, through a fine stainless steel tube into the atrium.

MEASUREMENT OF FLOW VELOCITY AND TURBULENCE The flow velocity distal to the valve was measured by means of a Disa conical hot film anemometer probe and its associated anemometer, linearizer, and other electronic equipment. The hot film anemometer is the best instrument so far developed for analysing velocity microstructures of turbulent liquid flow. The principle of operation is based on measurement of the conductive heat loss from a heated sensing element to the surrounding liquid. The sensor is placed on the tip of the probe, from which a cable goes to the anemometer, consisting of a Wheatstone bridge in which the sensor forms part of one bridge arm, and an amplifier. The amplifier output voltage is fed to the bridge as its supply and also provides a measure of the heat loss of the sensor. The use of a linearizer enables a signal directly proportional to a liquid velocity to be obtained. The frequency response of the system is up to several kilo Hertz. The probe was situated on the horizontal axis of the ventricle and could be moved across the ventricle, from the centre line to the wall opposite the outflow tract. It could also be moved towards or away from the atrioventricular junction.

The output from the anemometer was connected to the linearizer. The linearized signal passed through the auxiliary unit and into a variable active filter, where the signal could be separated into various selected frequency bands. The output from the variable active filter was fed to a digital voltmeter, in order to measure the mean DC level of the signal, and to a root mean square voltmeter to read the low frequency fluctuating and higher frequency turbulence components of the signal.

\section{CONCLUSIONS}

The establishment of a reliable method of testing mitral valve prostheses is clearly long overdue. The method should produce results which are repeatable over long time intervals and when carried out in different units. Valve prostheses put forward for clinical use have presumably all undergone some degree of physical testing before being implanted in animals or patients. In many cases, however, we could not find out exactly what tests had been done or what results had been obtained. Often, even when results are reported the conditions of testing are either not described or are so poorly controlled that evaluation is not possible. Pressure gradients for particular valves are often reported as a result of cardiac catheterization in the living patient. This not only exposes the patient to discomfort and even danger to discover facts that could be obtained with $a$. rig, but the difficulty in assessing cardiac output at the moment the gradient is measured makes the result of less value than that obtained with the rig.

We like to find out as much as possible about the behaviour of the valve before we test it biologically. We recognize that some properties of the valve can only be ascertained by their behaviour under biological test, but we still prefer 
to determine all those factors that can be measured by physical methods. The description of our apparatus and technique will, we hope, suggest a minimum standard of investigation that should be applied to any valve before it is considered for implantation. In a subsequent paper we hope to show some of the defects that our tests have revealed in valves in current use and the close correlation between our findings and clinical results.

The authors would like to thank the British Heart Foundation for a grant which enabled this work to be undertaken, and Drs. B. T. Bellhouse and D. L. Schultz, of the Department of Engineering, University of Oxford, for their comments on hot film anemometry.

\section{REFERENCES}

Barnard, C. N., Goosen, C. C., Holmgren, L. V., and Schrire, V. (1962). Prosthetic replacement of the mitral valve. Lancet, 2, 1087.

Beeson, J. K., West, A., and Burns, W. H. (1965). Engineering analysis of prosthetic heart valves. Circulation, 32, Suppl. 2, p. 46.

Calvert, G., Drabble, J., Serafin, R., and Temple, L. J (1964). An aortic pulse duplicator of simple design J. thorac. cardiovasc. Surg., 47, 633.
Cartwright, R. S., Palich, W. E., Ford, W. B., Giacobine, J. W., Zubritsky, S. A., and Ratan, R. S. (1962)ס Combined replacement of aortic and mitral valves $\delta$ An original transatrial approach to the aortic valve $\overline{\bar{e}}$ J. Amer. med. Ass., 180, 6.

Duran, C. G., Gunning, A. J., and McMillan, T. (1964)尺 A simple versatile pulse duplicator. Thorax, 19, 503

Geigy, J. R. (1956). Documenta Geigy-Scientific Tables, 5th ed., p. 304. J. R. Geigy, S.A., Basle.

Henderson, Y. (1906). The volume curve of the ventricles. of the mammalian heart, and the significance of thisw curve in respect to the mechanics of the heart-beat an the filling of the ventricles. Amer. J. Physiol., 16, 325

McDonald, D. A. (1960). Blood Flow in Arteries, pp. 38-41 Arnold, London.

Smeloff, E. A., Huntley, A. C., Davey, T. B., Kaufman, B. $\dot{\infty}$ and Gerbode, F. (1966). Comparative study of pros thetic heart valves. J. thorac. cardiovasc. Surg., 52, 841 인

Starr, A. (1960). Total mitral valve replacement; fixationand thrombosis. Surg. Forum, 11, 258.

Steinmetz, G. P., Jr., May, K. J., Mueller, V., Anderson? H. N., and Merendino, K. A. (1964). An improve accelerated fatigue machine and pulse simulator foc testing and developing prosthetic cardiac valves $J$. thorac. cardiovasc. Surg., 47, 186.

Taylor, M. G. (1959). The influence of the anomalous vis cosity of blood upon its oscillatory flow. Phys. in Med Biol., 3, 273.

Wieting, D. W. (1968). A method for analysing the dynamic flow characteristics of prosthetic heart valves. American Society of Mechanical Engineers, 68-WA/BHF-3. 